\title{
Specialist pride
}

\section{Shinji Tanigaki ${ }^{1}$}

(c) The Japan Society of Ultrasonics in Medicine 2020
It has long been said that ultrasonography is like having a second set of eyes for obstetricians. Use of transperineal ultrasonography is becoming more and more common in recent years for assessing labor progression in place of the conventional pelvic examination, which had been considered the best approach by far. It has gotten to the point where ultrasonography is now the first set of eyes for obstetricians. Ultrasonography has become an indispensable part of obstetrics and gynecology, but what about the position of the Japan Society of Ultrasonics in Medicine (JSUM) as a group of specialists, and JSUM-certified ultrasound specialists, in obstetrics and gynecology?

First, let us consider fetal ultrasound screening and JSUM. The goal of fetal ultrasound screening is achieved if it detects a fetus with a finding that suggests a disorder for which the prognosis can be improved with perinatal management and the mother is referred to an institution with a higher level of care. A screening examination needs to be efficient, simple, and objective. One of the foundations of obstetric care in Japan is the Guidelines for Obstetrical Practice, in which ultrasonography is divided into "fetal ultrasound" for detecting fetal structural anomalies and "regular ultrasound" for general examinations of pregnant women. The Guidelines also provide a recommended checklist for screening of fetal structural anomalies.

Unfortunately, however, the number of items on this recommended checklist has been reduced over the years, especially with respect to the fetal heart, leading to discrepancies with Level I procedures, which involve screenings performed by obstetricians, in the Fetal Echocardiography Guidelines published by the Japanese Society of Fetal Cardiology. Prioritizing simplification to promote more widespread use of screening and save limited perinatal health resources may be unavoidable, but as an ultrasound specialist, it leaves me

Shinji Tanigaki

tanigaki@ks.kyorin-u.ac.jp

1 Department of Obstetrics and Gynecology, Kyorin

University School of Medicine, Tokyo, Japan with a sad feeling. In the Guidelines for Obstetrical Practice, regular ultrasound and fetal ultrasound differ greatly, and the need for special training for operators is described. The level of medical care that is required and the medical needs of patients are rising. I feel like now more than ever, we need the guidance of JSUM.

Next, let us consider ultrasound specialist accreditations for obstetricians and gynecologists. Today, several fetal assessments are performed in the early stage of pregnancy. As a rule, clinical genetic-related accreditation and ultrasonic assessment-related international accreditation (e.g., Fetal Medicine Foundation, Nuchal Translucency Quality Review) are required before performing a fetal assessment. The fact that those who have undergone proper training perform the assessments, including counseling, and the fact that their precision is periodically assessed, should be lauded. How about ultrasound specialists? I assume they have all received the proper training. There are not many accreditations that require learning the basic principles of ultrasonography. Moreover, knowledge of not only obstetrics and gynecology but also a wide range of clinical disciplines is required to become a JSUM-certified specialist. I dare say that JSUM certification is broader in its scope than any other certification. Ultrasonography for fetal cardiac diseases is covered by health insurance at a limited number of institutions only. I hope to make the most of my JSUM certification with pride going forward.

Today, use of ultrasound has become commonplace, making it the perfect time for JSUM and JSUM certification to play a leading role. I believe that leadership will make JSUM an even more appealing organization and propel it forward. As a member of JSUM myself, I will make an effort to improve JSUM and the JSUM certification system.

Publisher's Note Springer Nature remains neutral with regard to jurisdictional claims in published maps and institutional affiliations. 